\title{
Efecto de la Deshidratación Osmótica y la Temperatura sobre el Color y la Fuerza Máxima de Fractura de Rodajas de Yuca en Freído por Inmersión
}

Fabián A. Ortega-Quintana*, Everaldo J. Montes-Montes, Omar A. Pérez-Sierra y Gabriel I. Vélez-Hernández Universidad de Córdoba, Facultad de Ingenierías, Grupo de investigación GIPPAL, Carrera 6 No. 76-103, Montería, Córdoba, Colombia. (e-mail: fortega@correo.unicordoba.edu.co)

${ }^{*}$ Autor a quien debe ser dirigida la correspondencia

Recibido Mar. 13, 2018; Aceptado May. 23, 2018; Versión final Jul. 27, 2018, Publicado Feb. 2019

\begin{abstract}
Resumen
Se estudia el efecto de la deshidratación osmótica y la temperatura de freído sobre el color y la fuerza máxima de fractura de rodajas de yuca ICA-costeña durante el freído por inmersión. Las rodajas de yuca fueron deshidratadas en solución de cloruro de Sodio al $3 \% \mathrm{p} / \mathrm{p}$, con temperatura controlada a $30^{\circ} \mathrm{C}$, por 4 horas y relación muestra/solución 1:20. El freído se realizó en aceite de palma a 140, 160 y $180^{\circ} \mathrm{C}$ durante un tiempo de 240 segundos. El color se determinó por Visión Computacional y la fuerza máxima de fractura fue medida en un analizador de Textura Shimadzu EZ Test ${ }^{\circledR}$. La temperatura de freído afectó significativamente $(p<0,05)$ los cambios de color y la fuerza máxima de fractura de la rodaja de yuca, mientras que la deshidratación osmótica sólo afectó significativamente $(p<0,05)$ a la fuerza máxima de fractura de la rodaja de yuca.
\end{abstract}

Palabras clave: freído; yuca; textura; color

\section{Effect of Osmotic Dehydration and Temperature on Color and Fracture Maximum Force of Yucca Root Slices in Deep-Fat Frying}

\begin{abstract}
The effect of osmotic dehydration and frying temperature on the color and fracture maximum force of yucca root slices, ICA-costeña variety, during deep-fat frying is studied. The yucca root slices were dehydrated in a solution of sodium chloride $3 \%(\mathrm{w} / \mathrm{w})$. The dehydration process took four hours, the temperature was set to $30^{\circ} \mathrm{C}$ and the ratio sample/solution was set to $1 / 20$. The frying was performed in palm oil at 140,160 and $180^{\circ} \mathrm{C}$ for 240 seconds. The color was determined by Computational Vision and the fracture maximum force was measured in a texture analyzer Shimadzu EZ Test $\AA$. It was found that the frying temperature had significant effect $(p<0,05)$ on the color changes and the fracture maximum force of the yucca root slice. The osmotic dehydration, on the other hand, significantly affected $(p<0,05)$ the fracture maximum force of the yucca root slice.
\end{abstract}




\section{INTRODUCCIÓN}

El freído por inmersión es una operación unitaria que involucra simultáneamente transferencia de calor y transferencia de masa y permite obtener productos con sabores, olores, colores y textura característicos. Las altas temperaturas que el freído maneja, entre 140 y $190^{\circ} \mathrm{C}$, influyen en el desarrollo de reacciones de pardeamiento no enzimático tales como la reacción de Maillard y en menor grado la caramelización de azúcares (Safari et al., 2018). Estas reacciones son responsables del desarrollo del color sobre la superficie del material en proceso. Paralelamente, se desarrollan gelatinización de almidones, desnaturalización de proteínas, pérdida de humedad y absorción de aceite, responsables en gran medida del desarrollo de la textura. Los cambios en la textura y en el color de la materia prima, reflejados en el producto final, serán percibidos por el consumidor quien emitirá un juicio sobre la calidad del producto y su aceptación o rechazo (Zhang et al., 2018; Zhang et al., 2016; Zhang et al. 2012; Pedreschi et al., 2006).

El desarrollo del color en las frituras comienza solamente si ha ocurrido una deshidratación en el alimento y depende de la tasa de secado y del coeficiente de transferencia de calor durante las diferentes etapas del freído. Se espera que las variables de proceso tales como tiempo, temperatura de aceite y tratamientos previos de las materias primas afecten al color de los productos fritos (Yang et al., 2016; Mery y Pedreschi, 2005). Los cambios del color en los tubérculos fritos son el resultado de la reacción de Maillard, la cual depende del contenido de los azúcares reductores (principalmente de D-glucosa) y los aminoácidos o las proteínas en la superficie, la temperatura superficial, el contenido de humedad y tiempo de fritura (GarcíaSegovia et al., 2016). A temperaturas menores de $60^{\circ} \mathrm{C}$, el pardeamiento es normalmente una reacción de orden cero, a temperaturas más altas se comportará como una reacción de primer orden. Un análisis de primer orden para la cinética del pardeamiento en el proceso de freído es esperado debido a que el proceso de freído por inmersión usualmente tiene un período muy corto con una temperatura en la superficie mayor a los $60^{\circ} \mathrm{C}$ (Medeiros et al., 2012; Zhang et al., 2012; Serpen y Gökmen, 2009).

Los cambios de textura, representados por la fuerza máxima de fractura, durante el proceso de freído son el resultado de muchos cambios físicos, químicos y estructurales producidos en el tejido, que también incluye transferencia de calor y de masa con reacciones químicas. En productos con contenido de almidón relativamente alto, tales como las papas fritas, la mayor influencia sobre la textura la produce la gelatinización del almidón durante el calentamiento (Mery y Pedreschi, 2005; Pedreschi et al., 2004). La evolución de la textura de tejidos vegetales durante el freído muestra una etapa inicial, en la cual el tejido se ablanda y comienza a cocinarse, y una etapa posterior en la cual la formación de la corteza inicia y progresivamente endurece. Un modelo empírico con la suma de dos términos ha sido propuesto para predecir los cambios de la textura, representada por la fuerza máxima de fractura: el primero para el ablandamiento con cinética de primer orden y el segundo para el endurecimiento con comportamiento lineal o cuadrático (Pedreschi et al., 2004; Pedreschi y Moyano, 2005; Moyano et al., 2007; Troncoso y Pedreschi, 2007). Por otro lado, la deshidratación osmótica ha sido reportada como un tratamiento que influye en la calidad final del producto, minimizando los cambios de color y mejorando las propiedades texturales del producto (Pedreschi y Moyano, 2005).

Para la yuca variedad ICA-costeña no existen investigaciones que hayan reportado la evolución durante el freído por inmersión del color y de la fuerza máxima de fractura y mucho menos modelado matemáticamente tales cambios. Por lo tanto, el objetivo de esta investigación fue estudiar el efecto de la deshidratación osmótica y la temperatura de freído sobre la cinética de los cambios de color y la cinética de la fuerza máxima de fractura desarrollada en las rodajas de yuca variedad ICA-costeña durante el freído por inmersión. Con los resultados obtenidos en esta investigación, se podrán implementar criterios de control de calidad del proceso de freído de esta variedad de yuca, adicionalmente con los datos experimentales presentados se tendrá una base de datos para utilizarse en la obtención de mejores modelos matemáticos que describan y expliquen mejor los cambios de color y fuerza máxima de fractura, ya que los modelos aquí propuestos son de carácter empírico o predictivos (Ortega y Montes, 2015).

\section{METODOLOGÍA}

La metodología se presenta en seis subsecciones: materia prima, deshidratación osmótica de la materia prima, proceso de freído por inmersión, medida del color de las muestras, medición de la fuerza máxima de fractura $\left(\mathrm{F}_{\mathrm{MAX}}^{\star}\right)$ y análisis estadístico.

\section{Materia prima}

La yuca (Manihot esculenta Crantz) variedad ICA-costeña se obtuvo del banco de germoplasma suministrado por CORPOICA (Montería, Colombia). El tubérculo se lavó, peló y cortó en rodajas con diámetros de 3,5 $\pm 0,03$ cm y 1,308 $\pm 0,05 \mathrm{~mm}$ de espesor. 


\section{Deshidratación osmótica de la materia prima}

Las muestras de yuca fueron deshidratadas osmóticamente en solución acuosa de cloruro de Sodio al $3 \%$ $\mathrm{p} / \mathrm{p}$, con temperatura controlada a $30^{\circ} \mathrm{C}$ en calentador modelo TECTRON-BIO-20 (marca registrada de Instrumentación Científica Técnica, S.L). El tiempo de deshidratación osmótica de las muestras y la relación muestra/solución fue de 4 horas y 1:20, respectivamente. El exceso de agua sobre la superficie del producto fue removido con papel absorbente.

\section{Proceso de freído por inmersión}

Las muestras deshidratadas osmóticamente fueron sometidas a freído por inmersión en aceite de palma marca CARIBE ${ }^{\circledR}$ (producido por FAMAR, industria colombiana) a temperaturas de 140,160 y $180^{\circ} \mathrm{C}$. El freído se llevó a cabo en una freidora marca DUPREÉ® de 2 litros de capacidad y con controlador de temperatura de $\pm 1^{\circ} \mathrm{C}$. La relación kilogramo de muestra de yuca por litro de aceite fue de 0,005 y las rodajas fueron freídas a diferentes intervalos de tiempo hasta un tiempo final de 240 segundos. El proceso de freído también fue realizado a muestras sin pre-tratamiento (muestras control) para comparación con las muestras osmodeshidratadas.

\section{Medida del color de las muestras}

La medida del color se realizó según la técnica de visión computacional, descrita por Mery y Pedreschi (2005), con los siguientes detalles de procedimiento. Adquisición de imagen. Se utilizaron lámparas fluorescentes con temperatura de color de $6500 \mathrm{~K}$ (marca Philips, luz del día natural, potencia de $18 \mathrm{~W}$ ). Se utilizó una Cámara Digital (marca Canon®, serie PowerShot A430, Tokio, Japón, con 4.0 Mega Pixeles de resolución). Las imágenes fueron capturadas con la cámara digital en su máxima resolución (pixeles: 2272 x 1704) y se almacenaron en un computador (Pentium Dual-Core). La cámara digital se configuró así: modo de operación: manual, velocidad ISO: 100, balance de blanco: Fluorescente H, distancia focal: 20,7 mm, zoom: 9, flash: off, apertura AV: f/8.0, exposición TV: 1/15 s, calidad: raw, y macro: on. La muestra se ubicó a $35 \mathrm{~cm}$ de distancia vertical de las lámparas y a una distancia vertical de $22,5 \mathrm{~cm}$ de la cámara digital.

Procesamiento de Imagen

La cuantificación del color se realizó en el espacio CIELAB 1976 y se utilizó el software Adobe Photoshop CS5® (Adobe Systems inc.). A partir de la imagen de la rodaja capturada en formato TIFF no comprimido se obtuvo el valor de la media y desviación estándar para cada uno de los valores $L, a$ y b. Los valores $L, a$ y $b$ obtenidos se convirtieron a valores $L^{*}, a^{*}$ y $b^{*}$ utilizando las siguientes transformaciones lineales propuestas por Yam y Papadakis (2004), donde: $L^{*}$ es la claridad, $a^{*}$ es la posición entre rojo y verde y b* es la posición entre amarillo y azul.

$$
\begin{aligned}
\mathrm{L}^{*} & =\frac{100}{255} \mathrm{~L} \\
a^{*} & =\frac{240}{255} a-120 \\
\mathrm{~b}^{*} & =\frac{240}{255} \mathrm{~b}-120
\end{aligned}
$$

Cálculos de los cambios de color. Con los valores $L^{*}, a^{*}$ y $b^{*}$ de cada muestra se calcularon los cambios de color utilizando la distancia euclidiana $\left(\Delta \mathrm{E}^{*}\right)$ existente entre dos puntos en un espacio tridimensional (Mery y Pedreschi, 2005; Zhang et al., 2012). Esta distancia se calculó mediante la ecuación (4):

$$
\Delta \mathrm{E}^{*}=\sqrt{\left(\Delta \mathrm{L}^{*}\right)^{2}+\left(\Delta a^{*}\right)^{2}+\left(\Delta \mathrm{b}^{*}\right)^{2}}
$$

Donde $\Delta \mathrm{L}^{*}=\mathrm{L}^{*}{ }_{1}-\mathrm{L}^{*} 2, \Delta a^{*}=\mathrm{a}^{*}{ }_{1}-\mathrm{a}^{*}{ }_{2}$ y $\Delta \mathrm{b}^{*}=\mathrm{b}^{*}{ }_{1}-\mathrm{b}^{*}{ }_{2}$

\section{Cinética del color en el freído}

Para obtener los parámetros cinéticos de cambio de color se utilizó el modelo cinético de primer orden descrito en la ecuación (5), (Mery y Pedereschi, 2005; Zhang et al., 2012):

$$
\frac{\mathrm{d} \Delta \mathrm{E}}{\mathrm{dt}}=-\mathrm{K}_{\mathrm{E}}\left(\Delta \mathrm{E}-\Delta \mathrm{E}_{\mathbf{e}}\right)
$$


Donde $\Delta \mathrm{E}$ es el valor del color con respecto al punto de referencia en la escala $\mathrm{CIELAB}, \Delta \mathrm{E}_{\mathrm{e}}$ es el valor del color en el equilibrio con respecto al punto de referencia en la escala CIELAB, $K_{E}$ es la constante de velocidad del color, t es el tiempo de freído (s). Al tiempo cero el color tiene un valor inicial $\Delta \mathrm{E}_{0}$, por tanto la ecuación (5) queda como lo expresa la ecuación (6). La relación entre KE, incluida en la ecn. (6) con la temperatura de freído (T) fue descrita usando una relación tipo Arrhenius.

$$
\frac{\Delta \mathrm{E}_{(\mathrm{t})}-\Delta \mathrm{E}_{\mathrm{e}}}{\Delta \mathrm{E}_{0}-\Delta \mathrm{E}_{\mathrm{e}}}=\mathrm{e}^{-\mathrm{K}_{\mathrm{E}} * \mathrm{t}}
$$

\section{Medición de la fuerza máxima de fractura $\left(F_{\text {MAX }}^{*}\right)$}

La fuerza máxima de fractura fue medida según la metodología descrita por Pedreschi y Moyano (2005). Se utilizó un equipo analizador de Textura marca Shimadzu EZ Test ® (Shimadzu Corporation, Tokio, Japón) en un ambiente a $25^{\circ} \mathrm{C}$. El valor de la fuerza máxima de fractura fue obtenido de las curvas Fuerza contra distancia usando el software RheoMeter® Versión 2.0.4. Las curvas Fuerza contra distancia fueron obtenidas a diferentes tiempos de freído montando la muestra sobre dos puntos soportes separados 0,03 m y utilizando un diámetro de vástago de ruptura de 0,002 m.

Modelamiento de la fuerza de fractura normalizada. Pedreschi y Moyano (2005) propusieron un modelo empírico para predecir los cambios de textura durante el freído usando el parámetro de fuerza máxima de fractura normalizada, $\mathrm{MF}^{\star}$, (valor de fuerza máxima de fractura, $\mathrm{F}^{\star} \mathrm{MAX}$, en un tiempo $t$ dividido por el valor de fuerza máxima de fractura al tiempo cero, $\mathrm{F}_{0}{ }^{*} \mathrm{MAX}$ ). La MF* describe el ablandamiento de los tejidos y el proceso de desarrollo de la corteza que el alimento sufre durante la fritura y representa la fuerza necesaria para romper la muestra. La ecuación (7) fue utilizada para describir la variación de $\mathrm{MF}^{\star}$ con el tiempo de freído:

$$
M F^{*}=\mathrm{e}^{-\mathrm{k}_{\mathrm{s}} * \mathrm{t}}+\mathrm{k}_{\mathrm{h}} \mathrm{t}^{2}
$$

En esta ecuación $\mathrm{MF}^{*}$ es la fuerza máxima de fractura normalizada; $\mathrm{k}_{\mathrm{s}}$ es la constante cinética de ablandamiento del tejido del alimento durante el freído $\left(\mathrm{s}^{-1}\right)$; $\mathrm{kh}$ es la constante cinética para el proceso de endurecimiento de la corteza durante el freído $\left(\mathrm{s}^{-2}\right)$ y t es el tiempo de freído (s). Las constantes cinéticas del modelo fueron relacionadas con la temperatura de freído (T) usando una relación tipo Arrhenius.

Análisis estadístico

En el experimento se utilizaron tres réplicas de los tratamientos y cinco repeticiones de las muestras para realizar la evaluación del color y la fuerza máxima de fractura. Para los análisis de varianzas, test de diferencias de medias (Tukey) y ajuste de regresiones lineales y no lineales, con niveles de significancia del 5\%, se utilizó el software Statgraphics Centurion XV (Statistical Graphics Corporation, Rockville, USA).

\section{RESULTADOS Y DISCUSIÓN}

Los resultados se presentan y discuten en forma separada en las siguientes subsecciones: cinética de los cambios de color y cinética de la fuerza máxima de fractura normalizada $\left(\mathrm{MF}^{\star}\right)$.

\section{Cinética de los cambios de color}

En las Figuras 1 y 2 se muestra la evolución de las rodajas de yuca (control y osmodeshidratadas) sometidas a freído por inmersión. Se evidencia el cambio en el color a medida que transcurre el proceso de freído y es más pronunciado para la temperatura de $180^{\circ} \mathrm{C}$. Estos cambios de color son producidos por las reacciones de pardeamiento no enzimático, las cuales son más rápidas mientras más altas son las temperaturas e incrementan en la muestra el contenido de compuestos cíclicos coloreados denominados melanoidinas (Serpen y Gökmen, 2009).

Los cambios de color se cuantificaron midiendo los parámetros $L^{*}, a^{*}, b^{*}$, los cuales se muestran en las Figuras 3, 4 y 5 . En la Figura 3 se observa que el parámetro de claridad $\left(L^{*}\right)$ disminuye con el incremento de la temperatura y el tiempo de freído debido a que la rodaja de yuca se oscurece. Este oscurecimiento se debe a que la yuca tiene componentes con el grupo amino (proteínas) y azúcares reductores que son los responsables de los cambios de color como producto de la reacción de Maillard, la cual es altamente dependiente de la temperatura, por lo tanto, las temperaturas más altas provocan mayor oscurecimiento de la rodaja. La disminución del parámetro $L^{*}$ durante el freído por inmersión es un cambio característico y ha sido reportado por otras investigaciones (Pedreschi et al., 2006; Pedreschi et al. 2007; Bunger et al., 2003; Ngadi et al., 2007). 


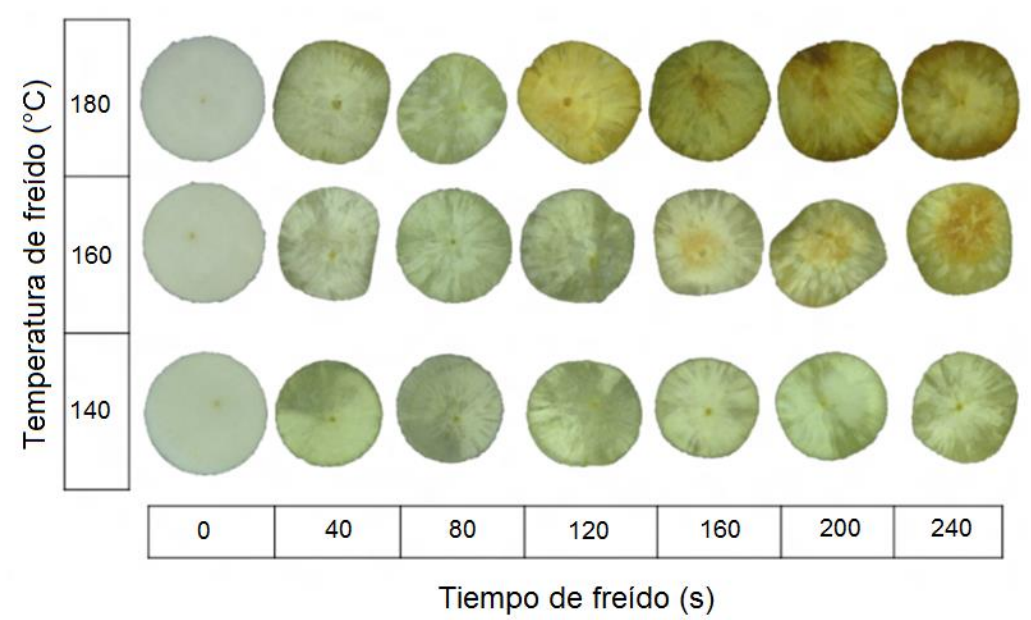

Fig. 1: Cambios de color en la muestra control.

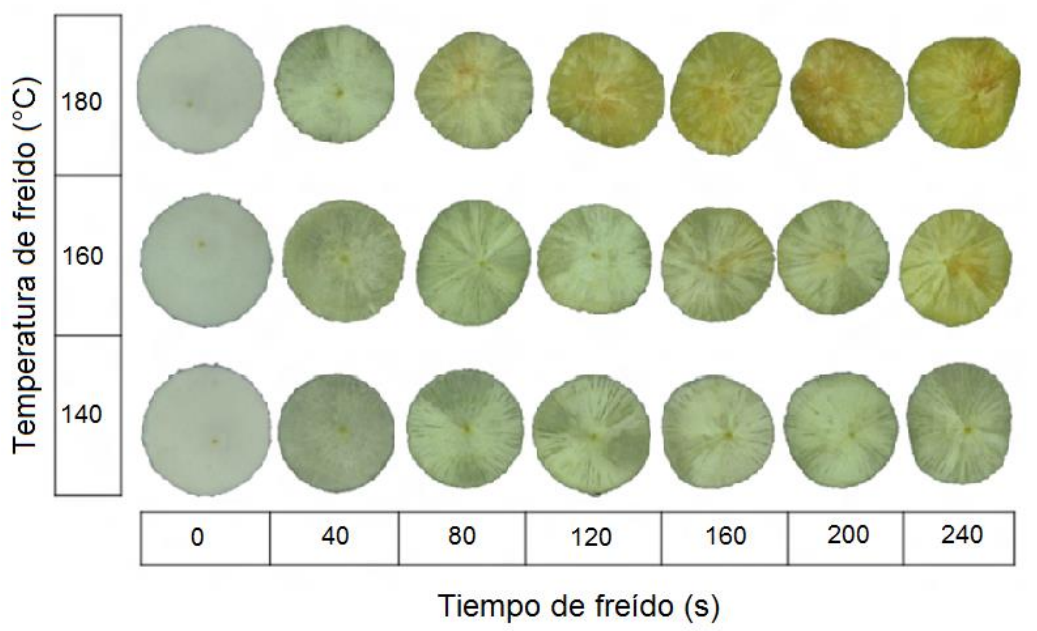

Fig. 2: Cambios de color en la muestra osmodeshidratada.

En las Figuras 4 y 5 se observa que los valores de los componentes cromáticos del color, $a^{*}$ y b*, incrementan con el tiempo de freído y la temperatura del aceite. El incremento es mayor cuando la temperatura del aceite aumenta debido a la formación de compuestos de la reacción no enzimática de Maillard y la caramelización de carbohidratos en la superficie, las cuales son más influyente en la muestra sometida a temperatura de freído de $180^{\circ} \mathrm{C}$. Estos resultados sugieren que las rodajas de yuca durante el freído tienden hacia el enrojecimiento y el color amarillo en su superficie. Este comportamiento presentado es semejante a lo que sucede en la muestra control y es corroborado por el análisis de varianza que arrojó que no existía diferencia significativa entre la muestra control y la muestra osmodeshidratada con respecto a los parámetros $L^{*}, a^{*}$ y $b^{*}$ (Pedreschi et al., 2006; Bunger et al., 2003; Romani et al., 2009).

La Figura 6 muestra el comportamiento característico de la cinética de primer orden. Al ajustar el modelo cinético de primer orden a los datos experimentales se encontraron altos coeficientes de determinación y una buena distribución de los residuos. Adicionalmente, se observa que los cambios de color final son mayores cuando la temperatura del aceite de freído es más alta, debido a que las altas temperaturas aumentan la velocidad de las reacciones de Maillard y caramelización de carbohidratos (Pedreschi et al., 2006; Bunger et al., 2003; Bunger et al., 2003; Romani et al., 2009).

La Tabla 1 muestra la dependencia que tienen los valores de $\mathrm{K}_{\mathrm{E}}$ con respecto de la temperatura de freído. Los datos de Muestra Control son tomados para comparación $(p<0,05)$ de Ortega y Montes (2015). Se observa que a mayor temperatura de freído es mayor la constante de velocidad de reacción del color $\left(\mathrm{K}_{\mathrm{E}}\right)$. Por otro lado, el análisis de varianza mostró que no existía diferencia significativa en los cambios de color entre la muestra control y la muestra osmodeshidratada. 


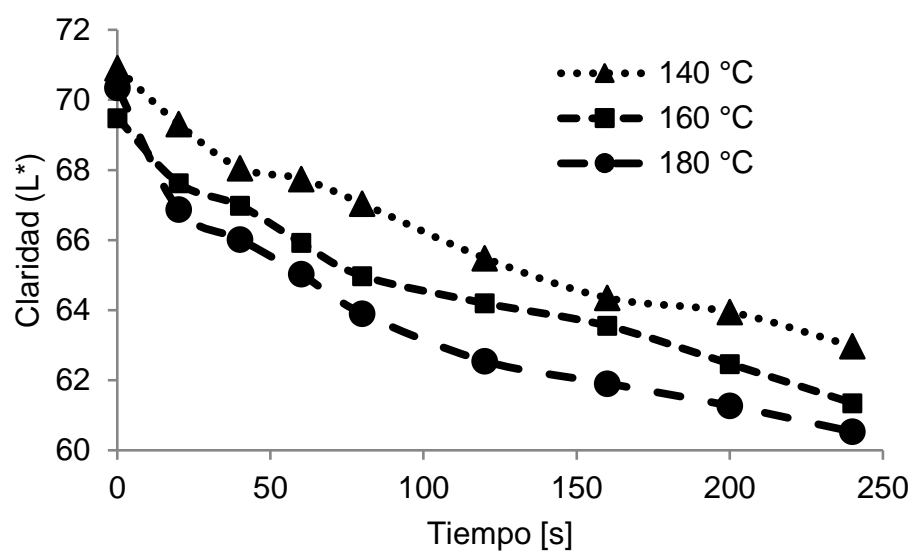

Fig. 3: Cambios en la Claridad $\left(L^{*}\right)$ durante el freído por inmersión de muestra de yuca osmodeshidratada.

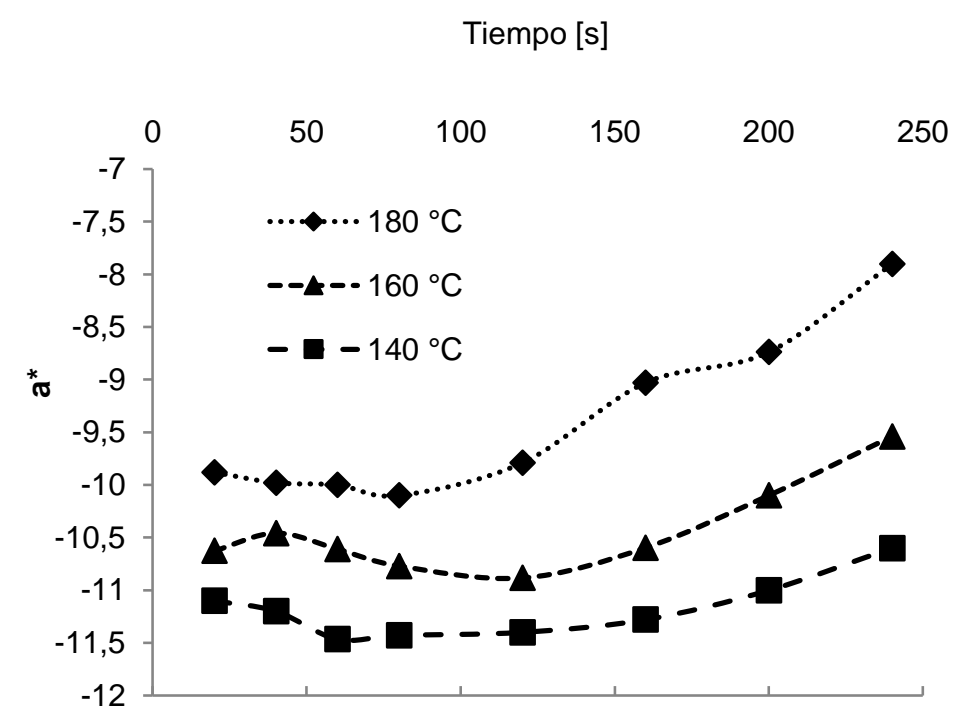

Fig. 4: Cambios en el parámetro $a^{*}$ durante el freído por inmersión de muestra de yuca osmodeshidratada.

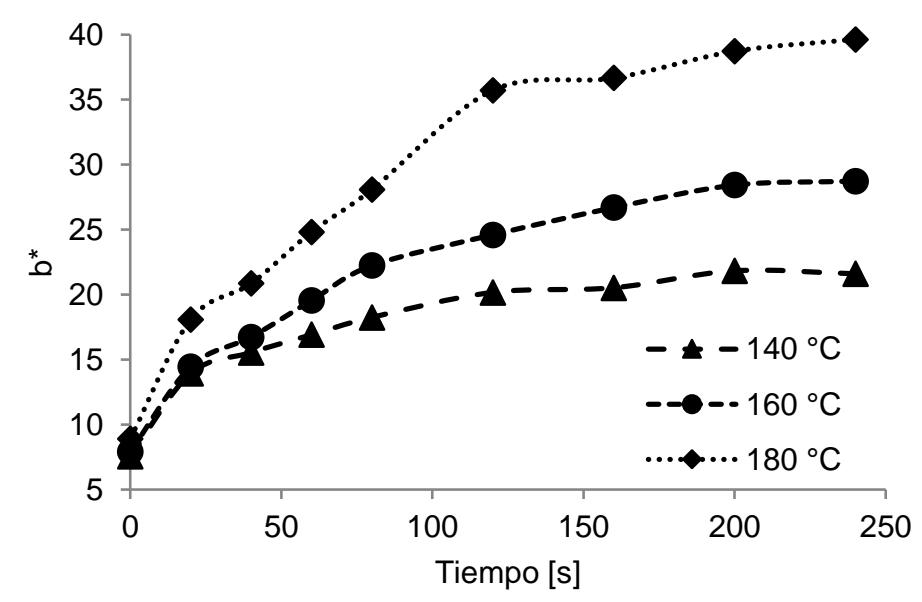

Fig. 5: Cambios en el parámetro $b^{*}$ durante el freído por inmersión de muestra de yuca osmodeshidratada. 


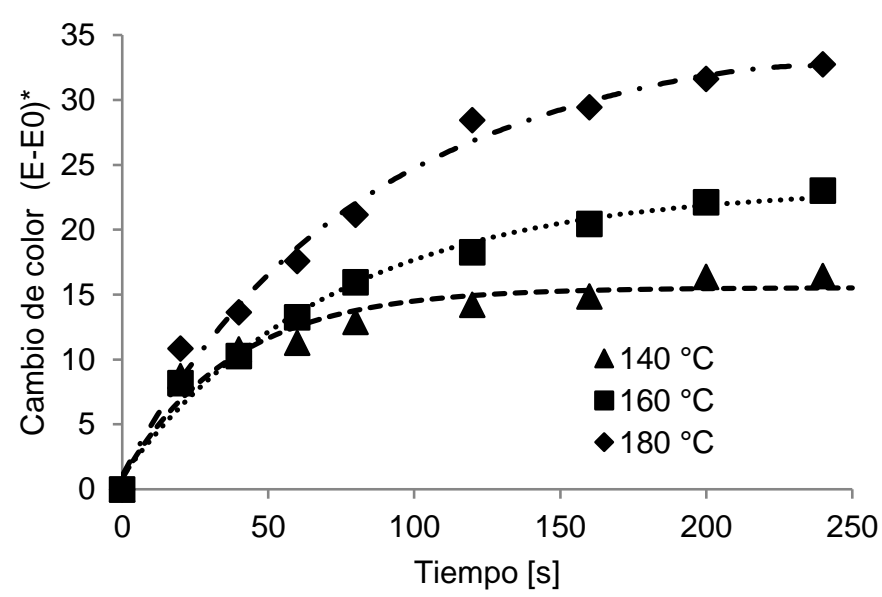

Fig. 6: Cambio de color durante el freído por inmersión en muestra de yuca osmodeshidratada.

Tabla 1: Constantes cinéticas del modelo de primer orden de cambio de color

\begin{tabular}{|c|c|c|c|}
\hline Muestra & $T\left({ }^{\circ} \mathrm{C}\right)$ & $K_{E} \times 10^{3}\left(\mathrm{~s}^{-1}\right)$ & $R^{2}$ \\
\hline \multirow{3}{*}{ Control $^{1}$} & $140^{\mathrm{a}}$ & $7,87 \pm 0,67$ & 0,979 \\
\cline { 2 - 4 } & $160^{\mathrm{b}}$ & $9,86 \pm 0,99$ & 0,990 \\
\cline { 2 - 4 } & $180^{\mathrm{c}}$ & $16,50 \pm 1,24$ & 0,988 \\
\hline \multirow{3}{*}{$\begin{array}{c}\text { Deshidratación } \\
\text { osmótica }\end{array}$} & $140^{\mathrm{a}}$ & $16,30 \pm 1,54$ & 0,976 \\
\cline { 2 - 4 } & $160^{\mathrm{b}}$ & $17,33 \pm 1,39$ & 0,981 \\
\cline { 2 - 4 } & $180^{\mathrm{c}}$ & $20,67 \pm 0,50$ & 0,998 \\
\hline
\end{tabular}

Los valores de la energía de activación para la constante cinética del modelo de primer orden de cambio de color para la muestra control y para la muestra osmodeshidratada fueron $28,61 \pm 2,66 \mathrm{~kJ} / \mathrm{mol}\left(\operatorname{con} R^{2}=0,939\right)$ y $8,41 \pm 1,82 \mathrm{~kJ} / \mathrm{mol}$ (con $\mathrm{R}^{2}=0,913$ ), respectivamente. Estos valores fueron más bajos que los valores típicos reportados para las reacciones de pardeamiento no enzimático que ocurren en alimentos y que están en el rango de 105 a $209 \mathrm{~kJ} / \mathrm{mol}$. Estas diferencias en los valores de energía de activación son debidas a los múltiples productos y a la complejidad de las reacciones que se presentan durante el proceso de freído, que hasta la fecha no han podido explicarse completamente (Pedreschi et al., 2006; Zhang et al., 2012; Bunger et al., 2003; Romani et al., 2009; Baik y Mittal, 2003; Vitrac et al., 2002).

\section{Cinética de la fuerza máxima de fractura normalizada $\left(M F^{*}\right)$}

El análisis de varianza mostró que la deshidratación osmótica de la yuca, la temperatura de freído, el tiempo de freído y la interacción pre-tratamiento-temperatura ejercieron un efecto significativo sobre la fuerza máxima de fractura normalizada. La Figura 7 muestra la fuerza máxima de fractura normalizada contra el tiempo de freído. Se observa que en los primeros instantes de freído se presentó una disminución de los valores de MF*, causada por la formación de gel de almidón contenido en la muestra y por la solubilización de los carbohidratos del tejido celular. Tiempo posterior, el valor de $\mathrm{MF}^{*}$ aumenta a causa del desarrollo progresivo y endurecimiento de la corteza de la rodaja de yuca por efecto de la disminución del contenido de humedad. En efecto, la Figura 7 muestra que las rodajas presentaron ablandamiento y endurecimiento más rápido del tejido a las temperaturas de freído más altas $\left(180\right.$ y $\left.160^{\circ} \mathrm{C}\right)$. Estos comportamientos también fueron reportados en investigaciones sobre el freído en rodajas de yuca escaldadas, en rodajas de papas y papas a la francesa, y se debe a la velocidad con que ocurre la pérdida de humedad, siendo más rápida a temperaturas altas (Pedreschi y Moyano, 2005; Troncoso y Pedreschi, 2007; Bunger et al., 2003).

En la Tabla 2 se muestran los valores de los parámetros cinéticos del modelo utilizado para ajustar los datos experimentales de la MF*. Los datos de Muestra Control son tomados para comparación $(p<0,05)$ de Ortega y Montes (2015). Los valores de $k_{s}$ y $k_{h}$ aumentan con la temperatura y son más grandes en las muestras con pre-tratamiento de deshidratación osmótica. 


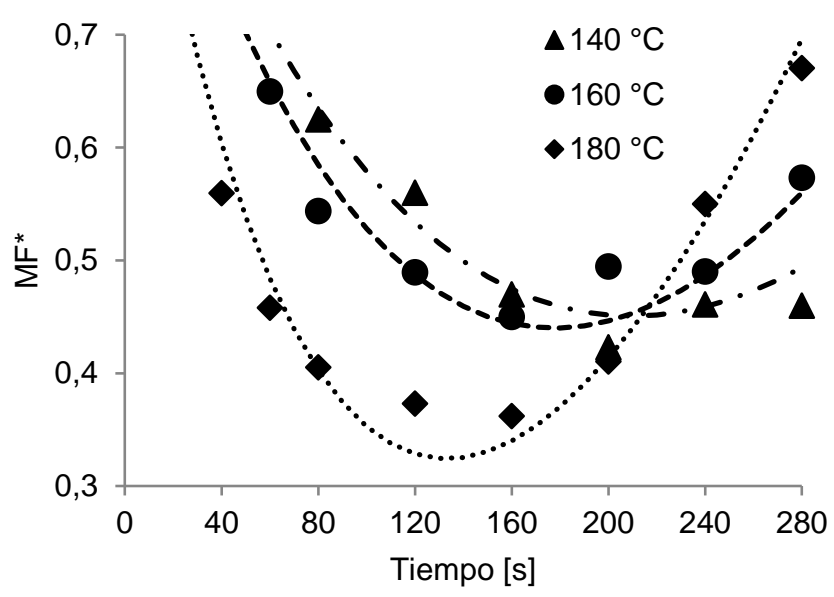

Fig. 7: Fuerza máxima de fractura normalizada $\left(\mathrm{MF}^{\star}\right)$ durante el freído de yuca osmodeshidratada.

Esto se debe al daño celular asociado al esfuerzo osmótico debido a la reducción de tamaño causada por la pérdida de agua, resultando en la pérdida de contacto entre la membrana celular externa y la pared celular de las células de yuca, lo cual lleva a cambios significativos en la arquitectura de los tejidos. Por otro lado, el valor de la energía de activación de la muestra control, 39,97 $\pm 2,31 \mathrm{~kJ} / \mathrm{mol}$ (con $\left.R^{2}=0,924\right)$, fue más alto que el valor de la energía de activación de la muestra osmodeshidratada, $29,16 \pm 1,81 \mathrm{~kJ} / \mathrm{mol}$ (con $R^{2}=0,909$ ). Los valores más altos de energía de activación en la muestra control indican que es más difícil la iniciación de los procesos de ablandamiento y endurecimiento de la corteza y se debe a que el tejido celular no ha sido modificado, lo cual si ocurre en la muestra osmodeshidratada (Pedreschi y Moyano, 2005; Rastogi et al., 2002).

Tabla 2: Constantes cinéticas $k_{s}$ y $k_{h}$ del modelo de fuerza máxima de fractura normalizada.

\begin{tabular}{|l|c|c|c|c|}
\hline Muestra & $T\left({ }^{\circ} \mathrm{C}\right)$ & $k_{s} \times 10^{3}\left(\mathrm{~s}^{-1}\right)$ & $k_{h} \times 10^{6}\left(s^{-2}\right)$ & $R^{2}$ \\
\hline \multirow{4}{*}{ Control $^{1}$} & $140^{\mathrm{a}}$ & $2,60 \pm 0,14$ & $2,12 \pm 0,23$ & 0,927 \\
\cline { 2 - 5 } & $160^{\mathrm{b}}$ & $6,11 \pm 0,33$ & $4,85 \pm 0,32$ & 0,922 \\
\cline { 2 - 5 } & $180^{\mathrm{c}}$ & $7,19 \pm 0,32$ & $8,77 \pm 0,34$ & 0,942 \\
\hline \multirow{3}{*}{ Deshidratación osmótica } & $140^{\mathrm{bd}}$ & $6,20 \pm 0,22$ & $4,05 \pm 0,27$ & 0,977 \\
\cline { 2 - 5 } & $160^{\mathrm{ce}}$ & $7,50 \pm 0,34$ & $5,58 \pm 0,35$ & 0,930 \\
\cline { 2 - 5 } & $180^{f}$ & $13,20 \pm 0,74$ & $8,56 \pm 0,20$ & 0,972 \\
\hline
\end{tabular}

\section{CONCLUSIONES}

La deshidratación osmótica no tuvo efecto significativo sobre los cambios de color, pero sí tuvo efecto significativo sobre los cambios de la fuerza máxima normalizada de la rodaja de yuca frita de la variedad ICAcosteña. La temperatura de freído tuvo un efecto significativo sobre los cambios de color y la fuerza máxima de fractura normalizada de la rodaja de yuca frita. Los cambios de color final son mayores cuando la temperatura del aceite de freído es más alta, por otro lado, a temperaturas de freído más altas las rodajas presentaron ablandamiento y endurecimiento más rápido del tejido y un valor más alto de la fuerza máxima de fractura normalizada. Los modelos propuestos se ajustan adecuadamente a los datos experimentales de los cambios de color y de la fuerza máxima de fractura normalizada durante la fritura de rodajas de yuca de la variedad estudiada.

\section{AGRADECIMIENTOS}

Los autores extienden su agradecimiento al laboratorio de Ingeniería Aplicada de la Universidad de Córdoba (Colombia) por el apoyo financiero del presente trabajo.

\section{REFERENCIAS}

Baik, O. y G. Mittal, Kinetics of tofu color changes during deep-fat frying, Lebensmittel-Wissenschaft and Technologie Journal, 36(1), 43-48 (2003)

Bunger, A., P. Moyano y V. Rioseco, NaCl soaking treatment for improving the quality of French-fried potatoes, Food Research International, 36(2), 161-166 (2003) 
García-Segovia, P., A. Urbano-Ramos, S. Fiszman y J. Martínez-Monzó, Effects of processing conditions on the quality of vacuum fried cassava chips (Manihot esculenta Crantz), LWT-Food Science and Technology, 69, 515-521 (2016)

Medeiros, R., F. Mestdagh y B. De Meulenaer, Acrylamide formation in fried potato products-Present and future, a critical review on mitigation strategies, Food Chemistry, 113, 1138-1154 (2012)

Mery, D. y F. Pedreschi, Segmentation of colour food images using a robust algorithm, Journal of Food Engineering, 66, 353-360 (2005)

Moyano, P., E. Troncoso y F. Pedreschi, Modeling texture kinetics during thermal processing of potato products, Journal of Food Science, 72(2), 102-107 (2007)

Ngadi, M., Y. Li e I. Oluka, Quality changes in chicken nuggets fried in oils with different degrees of hydrogenation, Lebensmittel-Wissenschaft and Technologie Journal, 40, 1784-1791 (2007)

Ortega, F. y E. Montes, Efecto del escaldado y la temperatura sobre el color y textura de rodajas de yuca en freído por inmersión, Revista ION, 28(1), 19-28 (2015)

Pedreschi, F., S. Segnini y P. Dejmek, Evaluation of the texture of fried potatoes, J. of Texture Study, 35(3), 277-291 (2004)

Pedreschi, F. y P. Moyano, Oil uptake and texture development in fried potato slices, Journal of Food Engineering, 70(4), 557-563 (2005)

Pedreschi, F. y P. Moyano, Effect of pre-drying on texture and oil uptake of potato chips, Lebensmittel-Wissenschaft und Technologie Journal, 38(6), 599-604 (2005)

Pedreschi, F., K. Kaack y K. Granby, Acrylamide content and color development in fried potato strips, Food Research International, 39(1), 40-46 (2006)

Pedreschi, F. y otros cinco autores, Color kinetics and acrylamide formation in $\mathrm{NaCl}$ soaked potato chips, Journal of Food Engineering, 79(3), 989-997 (2007)

Rastogi, N. y otros tres autores, Recent developments in osmotic dehydration: methods to enhance mass transfer, Trends in Food Science and Technology, 13(2), 48-59 (2002)

Romani, S. y otros tres autores, Image characterization of potato chip appearance during frying, Journal of Food Engineering, 93(4), 487-494 (2009)

Safari, A., R. Salamat y O. Baik, A review on heat and mass transfer coefficients during deep-fat frying: Determination methods and influencing factors, Journal of Food Engineering, 230, 114-123 (2018)

Serpen, A. y V. Gökmen, Evaluation of the Maillard reaction in potato crisp by acrilamide, antioxidant capacity and color, Journal of Food Composition and Analysis, 22(6), 589-595 (2009)

Troncoso E. y F. Pedreschi, Modeling of textural changes during drying of potato slices, Journal of Food Engineering, 82(4), 577-584 (2007)

Vitrac, O. y otros tres autores, Characterization of heat and mass transfer during deep-fat frying and its effect on cassava chip quality, Journal of Food Engineering, 53(2), 161-176 (2002)

Yam, K. y S. Papadakis, A simple digital imaging method for measuring and analyzing color of food surfaces, Journal of Food Engineering, 61(1), 137-142 (2004)

Yang, Y., A. Achaerandio y M. Pujolà, Influence of the frying process and potato cultivar on acrylamide formation in French fries, Food Control, 62, 216-223 (2016)

Zhang, Q. y otros tres autores, Chemical alterations taken place during deep-fat frying based on certain reaction products: A review, Journal Chemistry and Physics of Lipids, 165, 662-681 (2012)

Zhang, T. y otros tres autores, Effects of Initial Moisture Content on the Oil Absorption Behavior of Potato Chips during Frying Process, Food and Bioprocess Technology, 9(2), 331-340 (2016)

Zhang, Y.,y otros tres autores, The description of oil absorption behavior of potato chips during the frying, LWT-Food Science and Technology, 96, 119-126 (2018) 
